\title{
Energy from biomass: the potentialities, environmental aspects and technology
}

\author{
G. Genon, D. Panepinto \& F. Viggiano \\ Department of Environment, Land and Infrastructure Engineering, \\ Politecnico di Torino, Italy
}

\begin{abstract}
In the perspective of the production of energy and the satisfaction of different forms of energy requirements (electricity, thermal power, fuels), the possibility of using biomass in different forms is yet to be largely applied, but in the future there are very significant possibilities that this solution can be utilized a lot more. In fact it is important to consider these new possibilities in relation to the conversion of marginal agricultural areas to the production of energy crops, the destination of residues for treatment by conversion in energy factors (biogas, thermal gasification) or in energy (thermoelectric plants, co-generation, boilers), and new solutions for biofuels. With the aim to verify these aspects it is important to define the inventories of biomass that are at our disposition or that could be produced in the future, in connection with different hypotheses of development within this field. From these inventories it is possible to establish a potential percentage of the required energy that could be obtained from this type of feedstock. Besides the energy aspect, it is also necessary to evaluate the meaning, the positive aspects and the potential criticisms of these forms of energy production: these evaluations concern an assessment of the requirement for feedstock production, transport and transformation, the positive aspects of the right destination for residues, the effect on greenhouse production and climate change, the potential definition of environmental impacts originating from atmospheric emissions and residuals destination. It is also important to consider the consequences in terms of social and employment aspects; and obviously also aspects concerning the industrial costs, political prices for feedstock and fuels and carbon taxes. On the basis of the defined potential feedstock, it is necessary to evaluate the different technological schemes that can be utilised. In the present paper, all the previously indicated aspects will be reviewed, with reference to the actual and future situations in developed countries and in emerging economies.
\end{abstract}




\section{Introduction}

Looking at the present development of society and the instruments for improving quality of life, two of the most important problems here are the increase of energy requirements and the need to preserve the quality of the environment.

Concerning this first aspect, it is possible to distinguish a clear correspondence between an increase in production and capacity to satisfy fundamental needs of inhabitants and the quantity of energy that is continuously required; also if important efforts have been performed in order to obtain a decoupling between these two aspects, more generally and in different countries in different periods of time a general correlation between the development increase and the requirement of energy can clearly be observed [1].

The other aspect that has been indicated is the increasing concern with the reference to quality of the environment: in a global sense - climate change and general pollution, in local situations - the introduction of atmospheric pollutants, degradation of quality of natural waters and the over-exploitation of natural resource are quite general problems [2], that in each part of the world is on the table of operators and policy-makers.

In consideration of the strict correlation that can be observed between energy production and potential environmental impact [3], consideration of the possibility to use the resources of bioenergy seems to be a very important one.

The large and diffused presence of potential feedstock, the possibility to use energy systems in order to give a solution to the necessity of the disposal of residual organic flows and the positive aspects of limitation of some environmental impact phenomena are all conditions that suggest an increase in the utilisation of this form of energy. At the same time, in order to give the right dimension to the problem, it is required to verify what sort of capacity this form of energy could provide, to satisfy the local or medium range needs of energy, and to also consider any potentially critical consequences from the point of view of the environment, social development and regarding any ethical considerations.

In the present work a general analysis of these indicated phenomena will be presented, with particular reference to the European situation and with some specific case, which are useful in clarifying the pros and cons of this form of energy.

\section{Real bioenergy scenarios}

The definition of bioenergy in the general framework of world primary energy production can be considered very important [4] and in particular it is possible to observe the share of bioenergy between renewable energies $(77 \%)$ and the fundamental role that the production of woody biomass plays $(87 \%)$. With reference to the different origins of biomass, principal sources can correspond to specific agricultural products, residuals from zoo-technical activities and wood products.

The Technology Roadmap for Bioenergy for Heat and Power of IEA [4], where it is indicated that bioenergy accounts for roughly $10 \%(50 \mathrm{EJ})$ of the world's total primary energy supply, observes that most of this is corresponding 
to production with traditional schemes in non-OECD countries; on the contrary, in OECD countries bioenergy supply uses mainly modern technologies and overall plays a considerably smaller role than in developing regions.

Most bioenergy is currently consumed in the building sector, chiefly in Asia and Africa, where the traditional use of biomass for heating purposes is associated with very low efficiencies (10-20\%) and with significant health impacts originating from smoke pollution. It is also necessary to consider the potentially negative aspects of the production and use of biomass as energy in consideration of the competition with other biomass use, or with aspects of soil degradation and deforestation.

On the contrary, in OECD countries bioenergy plays a minor role in building, while there is an importantly increasing trend for co-firing or dedicated biomass co-generation power plants, however in this case, aspects of potentially local impacts and concerns about land use also have to be considered.

Energy supply from bioenergy corresponds today to $1.5 \%$ of world electricity production [4], with a global capacity of $280 \mathrm{TWh}$; this activity is chiefly concentrated in OECD countries, but China and Brazil are also becoming important producers, thanks to the promotion of electricity production from agricultural residues.

In general, many countries support policies that are important drivers for the development of the modern bioenergy supply chain, taking into account in any case the limits of feedstock costs and the sustainability of bioenergy.

In comparison with these considerations, an important increase in the use of bioenergy can be forecasted for the future; a hypothesis can be taken from [5], with reference to the technical and sustainable potential of biomass supply and the expected demand for biomass in 2050. Current world biomass use and primary energy demands are shown for comparative purposes. The meaning of this prediction and the significance of bioenergy in a general world energy scenario can be clearly evaluated.

Concerning the origins of biomass, the residues from forests, wood processing and food crops dominate [6], but also short-rotation energy crops currently provide a significant part of the total consumed biomass resource. In consideration of the growing demand for bioenergy in some regions compared to excess production in others, there is an international trade in solid and liquid biomass (pellets, biodiesel and ethanol).

\section{Future scenarios and inventories for biomass and connected possibilities}

The future for bioenergy is fundamentally connected with the availability of sufficient biomass for different productions (biofuels or thermal or electric power), and very strictly with the use of soil, taking into account strong competition with food production and industrial non-energy sectors (pulp and paper, building materials).

The fundamental aspects that must be considered are as follows:

- There is a large possibility for an increase of bioenergy by utilisation of energy crops in different types of land (there is plenty of surplus land 
that could be converted into energy crop plantations), but competition with other land uses and the potentially complex process for the creation of high efficiencies for these feedstock must be carefully considered;

- Large volumes of residual organic wastes can be valorised chiefly by their transformation into gaseous fuels (biogas for direct use, biomethane to be traded), but the compatibility of these valorisation technologies must be taken into account;

- Forest biomass is a traditional, well consolidated source for thermal energy and an increase in its utilisation for co-generation can be forecasted, but the notion of the forest as a carbon sink inevitably leads to the limitation of improvement in this sense and also sparks concerns about biodiversity.

On the basis of these considerations, an hypothesis for the global technical potential of land based biomass supply (primary energy) to the time frame of 2050 can be formulated, as in indicated in [5]; as it can be seen in this report, there is a very large difference between minimal and maximum hypotheses, but in any case an important increase in comparison with the real scenario can be easily observed.

In any case the limiting factor in bioenergy use is not resources, but accessibility, transportation costs and the resulting costs. It could be possible to satisfy $18 \%$ of the world's primary energy consumption in 2050 by woody biomass, but only with an extensive tax/subsidy policy in the energy markets, or by means of public investments in transport infrastructure and national forest programs.

In a paper concerning the potential use of bioenergy for the stabilisation of greenhouse gases (GHG) in the near future [7], it is estimated that the potential for this may be in the order of 0-150 EJ p.a. for residues, and that an increase from zero to several hundreds of EJ p.a. could be derived from energy crops, with a average number of around 100-200 EJ in 2050. The potential for bioenergy depends on a set of critical uncertainties (agricultural yields, dietary patterns, acceptability of use of natural areas for energy crops production and the possibility to use water-scarce and degraded areas).

\section{Technological consolidated and advanced schemes}

A schematic view of the wide variety of bioenergy routes can be found in fig. 1, taken from [4]: this gives an indication of the different types of feedstock that can be used, the conversion routes (comprehensive of pre-treatment and feedstock energetic valorisation), the final products in terms of produced heat or power and final products(chemicals or biofuels). It is important to note that in this scheme are presented and considered both consolidated, absolutely reliable but low-efficiency and in many cases pollutant solutions (as fuel wood direct combustion), and innovative, more energetically and environmentally promising schemes (like ligno-cellulosic second generation bioethanol, or gasification to co-generation). It is important to consider that, as reported in [8], concerning the 


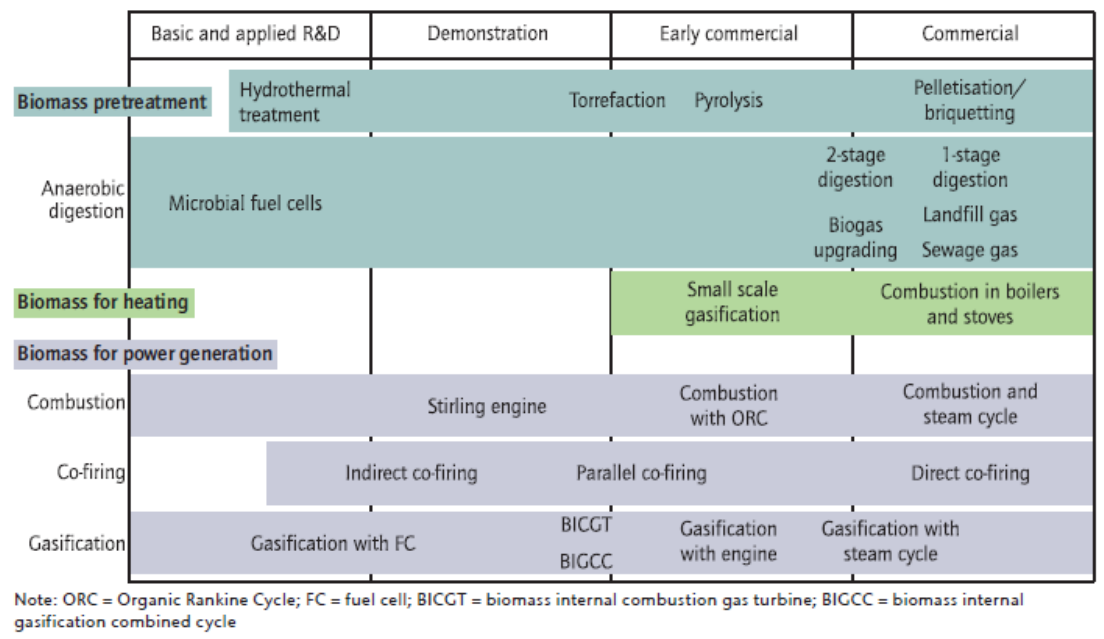

Figure 1: Overview of conversion technologies and their current development status.

examples of different biomass feedstock, there are different ranges of costs and plant capacities; and concerning the level of commercial exploitation, fig. 1 as previously indicated reports the most important routes.

Concerning the strict technological aspect, the following points can be considered:

- the direct biomass combustion for heating is a well developed technology, but it is frequently characterised by low efficiency and severe phenomena of smoke pollution; modern technologies like MSW incineration and the use of biogas can improve these critical aspects;

- biomass-based power plants present medium efficiencies (lower than fossil fuel thermoelectric plants) and also possibilities for co-firing in existing conventional plants must be considered;

- thermal gasification is a promising route, both from the point of view of efficiency and of containment of environmental impact, but the reliability of these plants still needs further verification;

- biorefineries can lead to the production of biofuels and other chemicals and in particular today there is an increasing interest in cellulosic ethanol plants where a higher compatibility, as we will see in the following chapters, can be observed;

- the technology of carbon capture and storage is applied in bioenergy conversion plants, leading to the removal of $\mathrm{CO}_{2}$ from the atmosphere and injection into a long term geological storage with a global negative emission;

- the bioenergy technologies for heat and power can lead to some efficiency improvement, but higher results can be forecasted from development solutions such as biomass pre-treatment and 
thermochemical gasification; also some innovation in the full scale exploitation of these systems is yet to be realised;

- improvement in small scale co-generation units and trigeneration technologies is another possibility to increase the competitive nature of these systems with conventional diffused systems for energy production, chiefly in developed metropolitan areas; also the aspect of the reduction of capital intensity for electricity production must be considered;

- the treatment of different type of wastes (incineration of MSW, anaerobic digestion of organic residues) requires, in account of the difficult characteristic of the treated incoming flux, very specific and costly technology and also high operating costs, but the credit from the right destination of wastes can represent an important benefit;

- process residues, such as black liquor for pulp and paper industry, bagasse from sugarcane and wood residues can also represent important high calorific value fluxes that can be used to produce heat or electricity;

- bio-methane obtained by different technologies is an important transformation product from biogas [9]; its purpose would be to use it as a substitute for other automotive fuels or imission into the grid for natural gas can represent a very efficient solution, which is useful for avoiding local impact phenomena.

\section{$5 \quad$ Industrial costs and policy aspects}

The costs for power production from biomass are dependent upon different considerations: the industrial costs in terms of plant and operation, market considerations for the original resource and taxes or incentives corresponding to the environmental considerations.

The plant costs, as indicated in [8] depend on the technology, the region of utilisation, the nature of feedstock, the requirement for pre-treatment and handling, the scale of operation and its effect on efficiency. As a general indication, there are estimated equipment costs for different systems documented in literature and also the capital cost breakdown is reported; as it can be seen in these indications, the capital costs are chiefly dependent on feedstock conversion technology and handling machinery, which are typical aspects of biomass power plants.

As regards to the operation and maintenance costs, the fixed part [8] can be estimated as a percentage ranging from $1 \%$ to $6 \%$ of the initial cost per year, while variable operation and maintenance costs (non biomass fuel costs, ash disposal, replacement, etc.) range from 3 to $5 \mathrm{US} \$ / \mathrm{MWh}$.

The feedstock costs, that can represent a very important part of the total cost of produced power, depend on the origin (the lowest costs for agricultural residues or waste materials), collection and transport; an indication, referred to in the European market [10], is reported; different values can be observed in non European countries. From the point of view of costs for transport from far 
regions, an improvement in pre-treatment technologies (chiefly torrefaction) could increase the energy density of feedstock and consequently lead to a reduction in transport costs.

It is also important to consider the different categories of feedstock; in this connection it is important to observe that, when the thermal treatment of biomass is used for the specific waste of a limited region or of an agricultural activity, this can lead to a very high reduction of feedstock costs corresponding only to the transport. Consequently, a limited scale of the plant in this sense is required, with a negative counter-acting aspect as regards to the efficiency of the plant.

Looking at the latter points, it is clear that biomass-based energy production is at the moment scarcely convenient in account of the higher feedstock cost, the limited efficiency and the elevated pre-treatment costs of biomass-based technologies in comparison with conventional fossil fuel solutions. An indication in this sense can be obtained from [4].

The answer to this diseconomy can be found, as it is indicated in [10], in the possibility to find emerging green power markets who will pay extra costs for this type of energy; different policies can be adopted to this aim, as indicated below:

- introduce a tax credit for the production of power from biomass, in consideration of a diminished environmental impact;

- introduce preferential prices for biomass from an agricultural origin, chiefly in the case of non-food competitive energetic solutions;

- $\quad$ use preferential solutions for dispatching of biomass renewable energy.

Some examples of these policies in different countries are yet to have been introduced, but for a more general development of biomass energy, a clear trend of these policies is required.

\section{Environmental benefits (climate change)}

One of the fundamental drivers in the implementation of bioenergy consists of its positive effect in GHG emission limitation, in account of the carbon balance of the whole utilisation of biomass to produce energy, taking into account all the phases of the cycle.

For the definition of this balance, it is necessary to take into account the following steps:

- definition of land use and the modification of it for the production of biomass; the differences between its capacity to act as a carbon sink for the original destination of land and the new destination must be considered;

- evaluation of GHG contribution from the phases of production and harvesting of biomass, chiefly with reference to the production and use of fertilisers; 
- pre-treatment, drying and the transformation of the harvested biomass that is required to produce the feedstock for the energy production (in this phase it is often necessary to consider the use of fossil fuels);

- thermal treatment of biomass and direct emission of $\mathrm{CO}_{2}$ (the possibilities for the options here regarding CCS are very interesting when considering the result of a negative emission of GHG and the absorption of gas from the atmosphere during photosynthesis without subsequent emissions),

- the destination of wastes and residues and their eventual impact in terms of GHG generation.

All the steps considered above must be evaluated with a complete LCA (Life Cycle Assessment); while many specific case results are present in literature, give specific results and show the significance of GHG emission limitations and benefits, the operation still requires a well defined and generally accepted operating procedure.

Some results, for the different cases of power production (heat and/or electricity) or biofuels utilisation are useful for indication, but it is very important to take into account that these results cannot be generalised, as they strictly depend on local production conditions, pre-treatment schemes, energy conversion efficiency, use of residuals and by-products.

By taking into account mass balances, it is possible to obtain an indication of GHG emission from different types of biomass, compared with gas or coal production, taking into account the strict emission from combustion and common agricultural practice; it is very evident that there are substantial advantages in comparison. But, if the impact from land use change is also taken into account, quite different results have been reported, especially in comparison with natural gas; it is clear that, depending on the original use of land, quite different situations can arise - from a very positive case where there is no land use change to quite a negative one, in the case of conversion from grassland, which has a high potential for carbon entrapment.

Concerning land use change and the effect on GHG emissions, the analysis that can be drawn from [11] indicates that a substitution of corn based ethanol to gasoline for automotive purposes leads to a limited reduction in GHG emissions (10-15\%); a similar approach, made by the UK Department of Transport [12], to the utilisation of some well established biofuels has led to similar results, with a conclusion leading to positive or negative land use change effects depending on the type of cultivation, the utilisation of by-products and the importance of integration with other agricultural activities. Indirect effects of land use change (corresponding to the displacement of original activities to other geographical situations as a consequence of the introduction of energy cultures) are well described in [13]. Taking also into account other GHG emissions and by using a complete carbon cycle, the GHG emissions from biofuels and the production of bioenergy are compared with conventional routes. An analysis to evaluate the indirect land use change and the effects of GHG emissions is reported in [14]. The tool of LCA, described in [15] is very useful for arriving at global results. 


\section{Environmental limits (local aspects)}

In comparison with the benefits concerning the limitation of GHG emissions and climate change, it is necessary to consider the consequences of bioenergy on biodiversity and natural resource management [16]; the trade-off between these two aspects (including those that are not directly comparable and chiefly directed towards differently exposed people and different forms of impact), is a fundamental aspect that is necessary to define the global sustainability of bioenergy in its different forms and different local applications.

One first aspect to take into consideration is the capacity of bioenergy to eliminate some forms of local impact, such as the wrong destination of agricultural or organic residues and the air toxic emissions from the combustion of fossil fuels; also the excessive use of fertilisers or chemicals for conventional or industrialised agricultural production can be limited.

On the contrary, potential critical aspects that must be considered are the effects on soil, water quality and availability and air quality.

The aspects of potential soil erosion must be properly addressed and the right choice made concerning the type and intensity of the cultivation of energy crops, which must be defined, chiefly by putting into operation the correct operation practices; the aspects of biodiversity, soil improvement and protection and the consideration of the natural destinations of soil and agricultural areas for food and natural activities must be taken into account.

As regards to water availability, it is necessary to consider the physical availability of water as well as the legal rights and access to water for cultivation and processing and the aspect of the final right destination of wastewater residual fluxes must be considered.

An important concern arises from the potential emission of pollutants from thermal use of biomass, chiefly for power production, in electricity or cogenerative assets; the formation of nitrogen oxides and coarse and fine dusts from biomass combustion is an important problem [17] that must be very carefully considered.

In order to limit this form of impact, there are two important tools that must be utilised; from one side it is necessary to use the best available technologies that are at disposition, as is indicated in [18], in order to limit the impact; performing solutions for the conventional pollution parameters are at disposition as the end-of-pipe measures to limit the emitted air pollution.

As a second tool, it is necessary to consider the environmental balance of a proposed bioenergy strategy (introduced pollutants in comparison with avoided pollutants arising from substituted forms of energy production) and to evaluate the consequences of this balance on the local air quality, as is indicated and described in detail in [19]; fig. 2 is an example of the maps of local air quality that can be calculated with predictive air pollution models and that are able to describe the presence or absence of the local effects of impact on the air quality.

In any case, the substitution of biofuels (bio-methane, second generation bio ethanol) to conventional fossil fuels seems to be a very interesting route for combining the positive effects as concerns to the limitation of GHG emissions and at the same time the absence of local effects on air quality deterioration. 


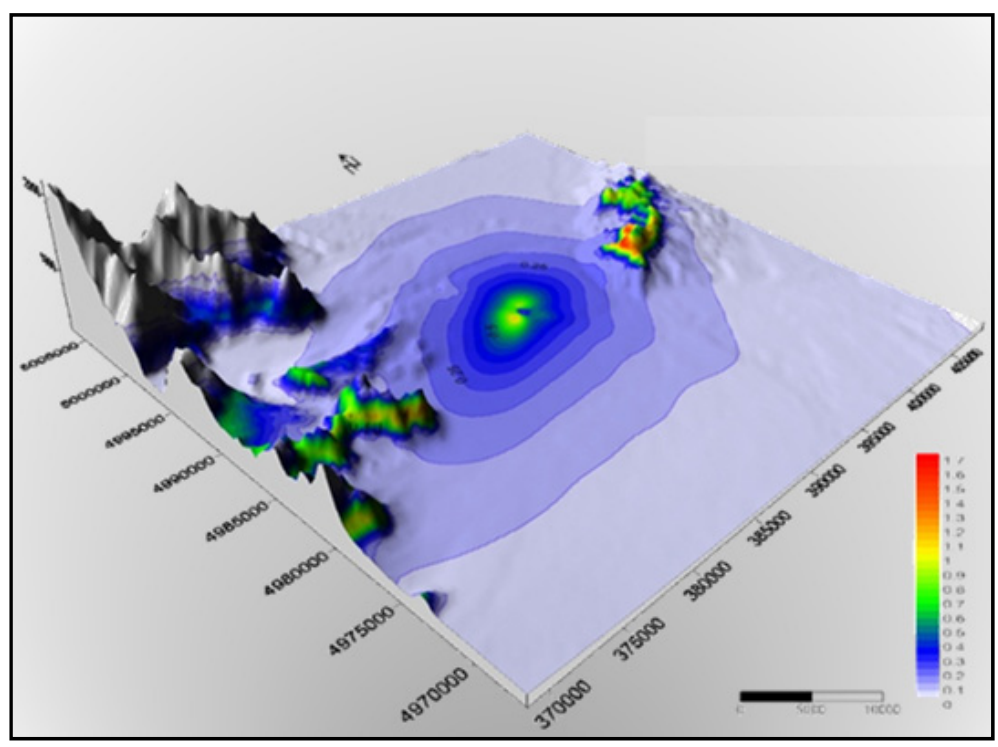

Figure 2: $\quad$ Example of a map of local air quality.

\section{Ethical and social considerations}

Alongside the considerations concerning the positive aspects of biomass in relation to the climate change benefits and the right evaluation of compatibility with reference to different environmental aspects, as it was indicated in the previous points, it is necessary to also evaluate the compatibility from the ethical and social point of view.

The principal aspects that must be considered are:

- the ability of modern bioenergy to provide safe and cheap energy services for the poorest populations: these possibilities, strictly required for an enhancement of living conditions of many countries, are connected with the definition of resource availability and the clarifying of competitive uses, economic access, reliability of solutions and financing;

- the consequences for agro-industrial development and job creation: the creation of small companies directly operating in the territory, the development of rural and transformation employment and the development of infrastructure are all very important implications for this type of development;

- improvement to the structure of agricultural activities, with the direct involvement of farmers in the production and use of biofuels, with particular emphasis on the second generation, avoiding competition with food products and consequent cost increases; capacity building is an important option for this improvement; 
- priority for food supply and food security: taking into account the fundamental priority of food satisfaction in under-developed areas, it is necessary to develop an analytical framework to fully understand the long term impacts of expanded bioenergy production; also an enhancement in agricultural productivity and sustainability, by conserving water and by improving soil fertility, is useful to diminish the competition of bioenergy;

- the diversification of global fuel and energy supply can have a positive effect on the global energy market, with a redistribution of producers and a lower risk of artificial cost structure; the trade policy of products would also be positively affected by this energy transformation;

- no additional negative biodiversity impacts: a correct feedstock choice and improvement of soil health in the framework of best land use can preserve biodiversity, minimise chemical use for fertilisation and reduce water needs; also the good management of residues and wastes can have positive effects on maintaining the health of the ecosystem.

\section{Conclusions}

On the basis of all the previous considerations, some indications for a useful enhancement of the application of bioenergy can be described, as in the following list of arguments:

- $\quad$ increase research efforts on the development of bioenergy feedstock;

- define the most promising locations for feedstock production and evaluate land suitability;

- $\quad$ increase efficiency of traditional biomass systems;

- $\quad$ support the research and the application of co-generation systems, on the small and large scale;

- define internationally agreed criteria for sustainability, in account of land use and utilisation;

- $\quad$ support international biomass trade with the introduction of technical standards;

- improve the collaboration on capacity building and technology transfer;

- remove inefficient fossil fuel subsidies and introduce prices for $\mathrm{CO}_{2}$ emissions;

- $\quad$ adopt measures for bioenergy development as feed-in tariffs, tradable green certificates and tax incentives;

- $\quad$ set minimum GHG reduction targets and introduce requirements in the national planning schemes for energy;

- improve infrastructures to transport feedstock at a reasonable cost with the minimum GHG emissions.

With the cooperation of intergovernmental organisations, development agencies, national and local authorities, universities and industries on these targets, it should be possible to develop a useful and sustainable model of development for this fundamental form of energy. 


\section{References}

[1] T.G. Gutowski et al. "The energy required to produce materials: constraint on energy intensity improvements, parameters of demand", Phil. Trans. R. Soc. 13 March 2013, vol 371, 1986;

[2] L.N. Lansal et al. "Scheme relationship for NO2 pollution and urban population size: a satellite perspective", Environ. Sci. Technol. 2013, 47 (14), 7855-7861;

[3] I. Dincer "Environmental impacts of energy", Energy Policy 27 (1999), 845-854;

[4] IEA Technology Roadmap: Bioenergy for heat and power, 2012;

[5] P. Lauri et al. "Woody biomass energy potential in 2050", Energy Policy, http://dx.doi.org/10.1016/j.enpol.2013.11.033;

[6] AEBIOM, European Biomass Association, European bioenergy outlook 2013;

[7] D.P. Van Vuuren et al. "Bioenergy use and low stabilisation scenarios" The energy journal, vol. 31 (special issue 1), 2010;

[8] IRENA, Renewable Energy Technologies: Cost Analysis Series, Biomass for Power Generation, Volume 1: Power sector issue 1/5, June 2012;

[9] J.R.H. Ross et al., The catalytic conversion of natural gas to useful products, Catalysis Today, 30 (1996), 193-199;

[10] Renewables 2013, Global Status Report, www.ren21.net;

[11] IEA Bioenergy, Bioenergy, Land use change and climate change mitigation, IEA Bioenergy: ExCo:2010:03;

[12] E4Tech, "A causal descriptive approach to modelling the GHG emissions associated with indirect land use impacts of biofuels", Final report, UK Department of transport, October 2010;

[13] R. Edwards et al., "Indirect land use change from increased biofuels demand" JRC European Commission, EUR 24485 EN-2010;

[14] Oiko Institut, The "iLuc Factor" as a means to hedge risks of GHG emissions from indirect land use change, Darmstadt, July 2010;

[15] F. Cherubini et al., "Energy and greenhouse gas-based LCA of biofuels and bioenergy system: key issues, ranges and recommendation", Resource, Conservation and Recycling 53 (2009), 434-447;

[16] Biofuels: ethical issues, Nuffield Council on Bioethics, London, April 2011;

[17] Sustainable Bioenergy: A framework for decision makers, UN-Energy, April 2007;

[18] European Commission, IPPC Reference Document on BAT for Large Combustion Plants, July 2006;

[19] D. Panepinto, G. Genon, E. Brizio, D. Russolillo. Production of green energy from co-digestion: perspectives for the Province of Cuneo, energetic balance and environmental sustainability, Clean Technologies and Environmental Policy, 2013; DOI: 10.1007/s10098-012-0568-0. 